\title{
Individual Differences in Sentence Comprehension: A Functional Magnetic Resonance Imaging Investigation of Syntactic and Lexical Processing Demands
}

\author{
Chantel S. Prat, Timothy A. Keller, and Marcel Adam Just \\ Carnegie Mellon University
}

\begin{abstract}
Language comprehension is neurally underpinned by a network of collaborating cortical processing centers; individual differences in comprehension must be related to some set of this network's properties. This study investigated the neural bases of individual differences during sentence comprehension by examining the network's response to two variations in processing demands: reading sentences containing words of high versus low lexical frequency and having simpler versus more complex syntax. In a functional magnetic resonance imaging study, readers who were independently identified as having high or low working memory capacity for language exhibited three differentiating properties of their language network, namely, neural efficiency, adaptability, and synchronization. First, greater efficiency (defined as a reduction in activation associated with improved performance) was manifested as less activation in the bilateral middle frontal and right lingual gyri in high-capacity readers. Second, increased adaptability was indexed by larger lexical frequency effects in high-capacity readers across bilateral middle frontal, bilateral inferior occipital, and right temporal regions. Third, greater synchronization was observed in high-capacity readers between left temporal and left inferior frontal, left parietal, and right occipital regions.

Synchronization interacted with adaptability, such that functional connectivity remained constant or increased with increasing lexical and syntactic demands in high-capacity readers, whereas lowcapacity readers either showed no reliable differentiation or a decrease in functional connectivity with increasing demands. These results are among the first to relate multiple cortical network properties to individual differences in reading capacity and suggest a more general framework for understanding the relation between neural function and individual differences in cognitive performance.
\end{abstract}

\section{INTRODUCTION}

Reading comprehension is a complex skill in which there are systematic individual differences in ability, even among college students. Proficiency differs at several levels of language processing. For example, good readers are faster and more accurate at word recognition (e.g., Bell \& Perfetti, 1994) and comprehension of syntactically complex sentences (e.g., Just \& Carpenter, 1992) than are poor readers. Individual differences in these abilities must ultimately be underpinned by individual differences in brain functioning. Our goal in the current study was to determine what facets of brain activity during sentence comprehension were related to behavioral measures of sentence comprehension skill.

Reprint requests should be sent to Chantel S. Prat, Center for Cognitive Brain Imaging, Carnegie Mellon University, Pittsburgh, PA 15213-3890, or via e-mail: csprat@andrew.cmu.edu. 
Neuroimaging research has provided important information about how various types of linguistic demands affect brain activity. Caplan and Alpert (1998), for example, found an increase in regional cerebral blood flow in the left pars opercularis as a function of increasing syntactic complexity. Keller, Carpenter, and Just (2001) extended these findings by investigating the relative effects of syntactic complexity and lexical frequency on neural processes by orthogonally manipulating the two variables. They found that syntactic complexity and lexical frequency manipulations independently caused increases in the traditional left perisylvian language areas. Additionally, they found an interaction between lexical frequency and syntactic complexity such that when the demand of both manipulations was high, extreme increases in activation were observed across a distributed network of language-related areas. The current study used functional magnetic resonance imaging (fMRI) to investigate individual differences in the neural responses to lexical and syntactic processing demands by studying readers with high or low language processing capacity, as indexed by the Daneman and Carpenter (1980) Reading Span Test (RST).

Individual differences in the ability to execute cognitive processes must ultimately be underpinned by individual differences in neural functioning, but it is unclear what facets of neural functioning are most closely related to behavioral measures. Our goal was to determine which properties of brain activation were associated with individual differences in comprehension ability. Newman and Just (2005) outlined a set of neural operating principles proposed to underlie "intelligent" or skilled cognitive functioning. They propose that intelligent behavior involves efficient use of neural resources, adaptability to changing demands, and coordination or synchronization of cortical networks. In the current article, we quantify neural efficiency, adaptability, and synchronization and examine them in high- and low-capacity readers to better understand the neural underpinnings of skilled language processing.

Most existing attempts to link individual differences in cognition with brain function have focused primarily on measures of neural efficiency. Maxwell, Fenwick, Fenton, and Dollimore (1974) were among the first to link cognitive abilities with neural efficiency. In an electrophysiological exploration of good and poor readers, they found evidence that good readers had more efficient neural processes (as indexed by lower power spectra in electroencephalogram) than did poor readers. Early positron emission tomography studies used glucose metabolic rates as indices of neural efficiency (e.g., Boivin et al., 1992; Haier et al., 1988). fMRI studies have attempted to measure neural efficiency in terms of the activation volume, which can be measured as the total number of voxels activated above some threshold value. Generally, the finding in both methodologies is that more proficient cognitive functioning in a given domain is associated with fewer activated voxels in a brain region that centrally participates in the processing in that domain (e.g., Newman, Carpenter, Varma, \& Just, 2003; Reichle, Carpenter, \& Just, 2000; Boivin et al., 1992; Haier et al., 1988). For example, in a sentence-picture verification task, participants with higher verbal abilities, as indexed by reading span scores, had lower activation volumes in typical language regions (e.g., Broca's area) when engaging in verbal strategies. Similarly, individuals with higher visualspatial skills, as indexed by mental rotation tasks, had lower activation volumes in typical visual association regions (e.g., parietal cortex) when engaging in spatial strategies (Reichle et al., 2000). The lower activation volume in more proficient individuals can be interpreted as using fewer neural resources to perform a given set of computations, and thus, can be thought of as neural efficiency. An individual's language capacity may therefore be correlated with indices of neural efficiency during sentence comprehension.

A second hallmark of an intelligent system is its ability to dynamically adapt to changing demands. Brain imaging studies provide evidence of such adaptation in terms of the activation of brain areas on an as-needed basis. Although a modal set of areas activates for any given task, additional areas may be recruited to deal with increasing demands. For example, Just, 
Carpenter, Keller, Eddy, and Thulborn (1996) found that as sentence complexity increases, right hemisphere homologues of typical left hemisphere language regions become activated. Existing evidence suggests that individual differences in intelligence may be linked to differential neural adaptability to task demands. Larson, Haier, LaCasse, and Hazen (1995) examined glucose metabolic rates as a function of task difficulty in individuals with high and average reasoning abilities. They found that individuals with high reasoning ability had higher metabolic rates during hard tasks, whereas individuals with average reasoning ability had higher metabolic rates during easy tasks. In an electrophysiological investigation of cognitive training, Neubauer, Grabner, Freudenthaler, Beckmann, and Guthke (2004) found that higherIQ individuals showed greater decreases in cortical activation as a function of training than did lower-IQ individuals. Therefore, comprehension ability may reflect differences in adaptability of an individual's neural network in the face of changing demands.

A third attribute of an intelligent system is the ability to coordinate the activities of its component subsystems. The various anatomical regions involved in processing a task must be able to effectively communicate and synchronize their processes for the system to function well. In a language task, this means that the areas responsible for executing subcomponent processes must collaborate to synthesize the information necessary for comprehension. Such collaboration may be reflected in functional neuroimaging studies by the correlation of the activation time series in a given region with the activation time series of another region. The extent to which the activation levels of two regions rise and fall in tandem is taken as a reflection of the degree to which the two regions are functionally connected, and the term that is widely used to refer to the activation time series correlation is functional connectivity (Friston, 1994). Although functional connectivity is a description of the synchronization of activation between remote cortical regions, it does not provide direct evidence that the activity of one region causes activity in another region, or that the regions are directly communicating. It, nevertheless, provides a useful characterization of brain activity at the network level. This level of description is particularly appropriate for evaluating the response of an intelligent system to task demands, and it may provide new insight into the nature of individual differences between such systems. Functional connectivity research has provided some evidence that as task demands increase, functional connectivity also increases, for example, as a function of working memory load (e.g., Diwadkar, Carpenter, \& Just, 2000), reflecting the need for tighter coordination in more demanding conditions. Functional connectivity has also been shown to increase with learning, at the same pace as the increase in performance, again indicating that the system coordination is an important facet of its effectiveness (Buchel, Coull, \& Friston, 1999). In a working memory task, Otsuka and Osaka (2005) found that younger individuals who perform better on the task had higher functional connectivity than older individuals who perform poorly on the task. It is possible that differences in comprehension ability may also reflect differences in functional connectivity between involved brain regions.

In this study, we compared neural efficiency (amount of activation), adaptability (changes in activation as a function of lexical frequency or syntactic complexity), and synchronization (correlation between time courses of activation for various regions in an active network) in high- and low-capacity readers, as indexed by the Daneman and Carpenter (1980) RST. Our goal was to discover the relation between characteristics of brain activation and the behavioral characteristics associated with high- and low-capacity readers.

\section{METHODS}

\section{Participants}

Forty right-handed, native English-speaking Carnegie Mellon undergraduate students participated in this experiment. All individuals were paid for their participation. The Daneman and Carpenter (1980) RST was administered to all participants. Data from 23 of the 40 
participants were reported in Keller et al. (2001) without regard to individual differences, and an additional 17 participants who met the selection criteria were recruited for this study. Only individuals receiving a score of 2.5 or below or 4.0 or above (approximately the top and bottom fifths of the population tested) were selected to participate in this study. Five of the 40 participants were excluded due to excessive head motion, leaving 18 who were classified as high-capacity (mean age $=21.6$ years, $S D=4.2$ years; mean reading $\mathrm{span}=4.4, S D=0.51,6$ men) and 17 who were classified as low-capacity (mean age $=22.4$ years, $S D=4.1$, mean reading span $=2.3, S D=0.26,13 \operatorname{men}^{1}$ ).

\section{Materials}

The study materials consisted of 80 sentences and comprehension probes used previously by Keller et al. (2001), who modified them from a set of sentences used by Just et al. (1996). They orthogonally crossed syntactic complexity (two-clause active-conjoined and object-relative sentences) and lexical frequency (high and low noun frequency) to form four experimental conditions, with 20 sentences in each condition. All sentences were similar in overall structure, consisting of two clauses and a final prepositional phrase. Sentences in the high-frequency condition contained nouns that occurred more than 70 times per million, according to Kucera and Francis (1967) word frequency norms, whereas sentences in the low-frequency condition contained nouns that occurred less than thrice per million. Half of the comprehension probes were true, and the other half were false. Half of the comprehension probes referred to information in the first clause of the sentence, whereas the other half referred to information in the second clause of the sentence. Sample stimuli appear in Table 1.

\section{Procedure}

All participants came in for behavioral testing and practice 1 to 2 days before their scan. Participants received the RST and the Edinburgh Handedness Inventory (Oldfield, 1971) prior to scanning.

During the fMRI scan, sentences were projected individually onto a plastic screen attached to the roof of the bore of the scanner. Participants viewed the sentences through a pair of mirrors attached to the head coil, with the display subtending a visual angle of approximately $30^{\circ}$. Participants were instructed to read each sentence for comprehension and to press a button when they were finished. The button press initiated presentation of the comprehension probe. Participants responded to the probe by pressing one of two buttons to indicate "true" or "false." Five sentences of the same type were presented sequentially in an epoch, and there were four epochs of each of the four conditions (sixteen total experimental epochs) with six baseline epochs interleaved. During the baseline epochs, participants were instructed to fixate on a centered " $X$ " for $24 \mathrm{sec}$. Epochs were separated from each other by $6 \mathrm{sec}$ of rest. Sentence reading time, probe reading and response time, and accuracy were recorded during the scan.

\section{fMRI Acquisition Parameters}

The imaging data were collected on a 3.0-T whole-body General Electric Signa scanner, retrofitted for echo-planar imaging (EPI), at the University of Pittsburgh Magnetic Resonance Research Center. Images were acquired using a GE quadrature birdcage head coil. A T2*sensitive gradient-echo EPI pulse sequence with $\mathrm{TR}=3000 \mathrm{msec}, \mathrm{TE}=25 \mathrm{msec}$, and a flip

\footnotetext{
${ }^{1}$ Because there were proportionately more men in the low-capacity group than in the high-capacity group, analyses of neural activation and synchronization were conducted using gender as a covariate. SPM analysis of brain activation revealed the same reading capacity effects reported in the manuscript when controlling for sex, although at a reduced significance level $(p=.003)$, presumably due to the large amount of shared variance between sex and reading capacity in this subject pool. Effects of sex were also observed (men > women in the supplementary motor area; women $>$ men in the bilateral superior temporal area), but did not overlap with the reading capacity results reported in this manuscript. There were no main effects of gender on region-of-interest (ROI)-based analyses of activation or neural synchronization.
} 
angle of $90^{\circ}$ was used to acquire functional images. Fourteen adjacent oblique-axial slices were acquired in an interleaved sequence, with $5 \mathrm{~mm}$ slice thickness, $1 \mathrm{~mm}$ slice gap, a $40 \times$ $20 \mathrm{~cm} \mathrm{FOV}$, and a $128 \times 64$ matrix size, resulting in an in-plane resolution of $3.125 \times 3.125$ $\mathrm{mm}$.

\section{DATA ANALYSIS METHODS}

\section{Behavioral Data}

Sentence reading times and error rates to comprehension probes were analyzed using 2 (reading capacity) $\times 2$ (syntactic complexity) $\times 2$ (lexical frequency) analyses of variance (ANOVAs). Reading capacity was a between-participants variable, and syntactic complexity and lexical frequency were within-participant variables. All effects were tested at a significance level of $p<.05$, unless otherwise indicated.

\section{Voxelwise Analyses of the Distribution of Activation}

The data were analyzed using SPM2 (Wellcome Department of Imaging Neuroscience; www.fil.ion.ucl.ac.uk/spm) to compare the distribution of activation for high-and low-capacity readers. Images were corrected for slice acquisition timing, motion-corrected, normalized to the Montreal Neurological Institute (MNI) template, resampled to $2 \times 2 \times 2 \mathrm{~mm}$ voxels, and smoothed with an 8-mm Gaussian kernel to decrease spatial noise. Statistical analyses were performed on individual and group data by using the general linear model as implemented in SPM2 (Friston et al., 1995). For individual participants, a fixed-effects model was used to estimate parameters and incorporated a high-pass filter with a cutoff of $512 \mathrm{sec}$ and an AR(1) correction for serial autocorrelation. Group analyses were performed using a random-effects model. Contrasts reflecting the group differences in the distribution of activation relative to fixation across conditions, the group differences in the syntactic effect (collapsing across lexical frequency conditions) and the group differences in the lexical frequency effect (collapsing across syntactic complexity conditions) were computed. Possible differences in deactivation (relative to fixation condition) were excluded from the analysis. Unless otherwise mentioned, a height threshold of $p<.001$ and an extent threshold of 6 voxels were used.

\section{Region-of-Interest Analyses of the Volume of Activation}

Four anatomical ROIs, originally defined by Tzourio-Mazoyer et al. (2002) in a cortical parcellation carried out on the MNI single-subject T1-weighted dataset, were selected in the left hemisphere and four in their right hemisphere homologues to encompass the main clusters of activation in both group activation maps across all sentence-minus-fixation contrasts. The four ROIs per hemisphere included inferior frontal (including the subregions of the pars opercularis and pars triangularis), temporal (including the superior and middle temporal gyri), parietal (including the subregions of the angular gyrus, posterior supramarginal gyrus, and intraparietal sulcus) and inferior occipital (inferior occipital gyrus). Figure 1 provides a schematic representation of the ROIs. The number of voxels activated in each ROI above the height threshold of $p<.05$, corrected for multiple comparisons, was calculated for each participant independently, for each of the four sentence conditions. The data for each ROI were analyzed separately using 2 (reading capacity) $\times 2$ (syntactic complexity) $\times 2$ (lexical frequency) ANOVAs. Reading capacity was a between-participants variable; Syntactic complexity and lexical frequency were within-participant variables. All effects were tested at a significance level of $p<.05$, unless otherwise indicated.

\section{Functional Connectivity}

The functional connectivity was computed separately for each participant as a correlation between the activation time courses (averaged over all of the activated voxels) in a pair of 
ROIs. The anatomical ROIs were the same ones used in the volume analysis described above, except that the pars opercularis was excluded from the frontal region, and the angular and supramarginal gyri were excluded from the parietal region because activation in these areas differed greatly between the high-and low-capacity groups. A subject was excluded from any analysis in which the number of voxels activated in either of the ROIs constituting the pair was less than 8 . The time course of signal intensity was extracted for each participant over the activated voxels within the ROI from the normalized and smoothed images that were low-pass filtered and had the linear trend removed. ${ }^{2}$ Fisher's $r$ to $z$ transformation was applied to the correlation coefficients for each participant prior to averaging and statistical comparison of the two groups. The data for each ROI pair were analyzed separately using 2 (reading capacity) $\times$ 2 (syntactic complexity) $\times 2$ (lexical frequency) ANOVAs. All effects were tested at a significance level of $p<.05$, unless otherwise indicated.

\section{RESULTS}

\section{Behavioral Data}

High-capacity readers read faster and were more accurate to the comprehension probes than were low-capacity readers, as shown in Figure 2. Analysis revealed a main effect of reading capacity for both sentence reading times $[F(1,33)=6.42, M S E=68,622,122]$ and response accuracies $[F(1,33)=5.60, M S E=0.13]$. High-capacity readers also made relatively fewer errors to the syntactically complex response probes than did low-capacity readers. Analysis of error rates revealed a reliable Reading capacity $\times$ Syntax interaction $[F(1,33)=6.1, M S E=$ $0.06]$.

All participants, irrespective of reading capacity, were slower and less accurate for syntactically complex sentences, and for sentences with low-frequency nouns. Analysis of sentence reading times revealed main effects of syntax $[F(1,33)=34.60, M S E=19,154,425]$ and frequency $[F(1,33)=83.53, M S E=25,498,345]$. This pattern was also observed in analysis of error rates [syntax: $F(1,33)=22.37, M S E=0.22$; frequency: $F(1,33)=4.13, M S E=0.02, p=.053$ ]. The sentence reaction times also showed a reliable Syntax $\times$ Frequency interaction $[F(1,33)=$ $12.54, M S E=2,789,199]$.

\section{Distribution of Activation}

The voxelwise analysis provided evidence that high-capacity readers had more efficient neural processes than did low-capacity readers. Specifically, high-capacity readers had reliably less activation in the bilateral middle frontal gyri, extending into the pars opercularis in the left hemisphere, and in the right lingual gyrus across experimental conditions than did low-capacity readers. Figure 3 depicts the activation maps for high- and low-capacity readers, as well as the reliable group differences. Note that the group differences seem to reflect additional activation in the language networks of low-capacity readers, rather than recruitment of additional areas. Although comparison of the within-group activation surface renderings in the top two rows of Figure 3 seems to indicate differences in left temporal activation between groups, these differences were not reliable. In fact, there were no areas in which high-capacity readers showed significantly greater activation than low-capacity readers across conditions.

\footnotetext{
${ }^{2}$ Because the duration of trials was dependent on the participant's reaction time, our original data analysis excluded images from the ends of longer-duration trials in order to equate the number of images used for calculating the functional connectivity across conditions and participants. It was noted, however, that low-capacity readers may be particularly affected by the truncation process, as their reading times were reliably longer, on average, than were high-capacity readers. At a reviewer's request, we conducted an additional analysis on the complete dataset. Our results were remarkably similar in the two analyses, with main effects of group reaching significance in the complete dataset and approaching significance in the truncated dataset. Only the analysis of the complete, untruncated dataset is reported in this manuscript.
} 
The voxelwise analyses also provided evidence for group differences in adaptability. Highcapacity readers showed a larger modulation of neural activation as a function of lexical frequency than did low-capacity readers in the bilateral middle frontal gyri, extending into the inferior frontal gyrus in the right hemisphere, in right temporal regions, and in the right caudate. Low-capacity readers, however, showed a greater lexical frequency effect in a small number of voxels in the superior medial frontal region. No group differences in neural adaptability as a function of syntactic frequency were observed. MNI coordinates, Brodmann's areas, and peak $T$ values for reliable group differences in activation from the voxelwise analyses are listed in Table 2.

\section{Volume of Activation}

The ROI-based analyses of the volume of activation provided additional evidence that highcapacity readers were more adaptable to lexical frequency demands than were low-capacity readers. The resulting Reading capacity $\times$ Lexical frequency interaction was reliable in the left temporal $[F(1,33)=4.85, M S E=204,115]$ and left inferior occipital $[F(1,33)=8.44, M S E=$ $505,112]$ regions, and approached significance in left inferior frontal $[F(1,33)=3.47, M S E=$ $262,244, p=.071]$ and right inferior occipital $[F(1,33)=4.07, M S E=206,449, p=.052]$ regions (see Figure 4). Follow-up analyses revealed that high-capacity readers showed a reliable increase in activation associated with decreasing lexical frequency in left inferior frontal $[F(1,17)=5.04, M S E=346,528]$ and inferior occipital $[F(1,17)=7.56, M S E=420,445]$ regions, whereas low-capacity readers did not [left inferior frontal, $F(1,16)=0.24$; left inferior occipital, $F(1,16)=2.02$. All readers showed greater activation for the low-frequency than the high-frequency condition in the left parietal region, resulting in a reliable main effect of frequency $[F(1,33)=4.94, M S E=156,914]$.

No group interactions with syntactic complexity were observed in the ROI-based analyses. Both groups activated a larger number of voxels in object-relative sentences than in activeconjoined sentences. This main effect of syntax was significant in left inferior frontal $[F(1,33)$ $=10.61, M S E=270,402]$, left temporal $[F(1,33)=17.29, M S E=317,275]$, and left parietal $[F(1,33)=16.85, M S E=239,720]$ regions. The mean number of voxels activated in high- and low-capacity readers for each sentence type appears in Table 3.

\section{Functional Connectivity}

High-capacity readers showed greater synchronization than low-capacity readers between key regions in the language network across conditions. This main effect of reading capacity was reliable for functional connectivity between left inferior frontal and left temporal regions $[F$ $(1,32)=5.13, M S E=1.42]$, between left temporal and left parietal regions $[F(1,32)=5.34$, $M S E=0.89]$, between left temporal and right inferior occipital regions $[F(1,32)=6.09$, $M S E=0.87]$, and approached significance between left temporal and left inferior occipital regions $[F(1,32)=4.07, M S E=0.95, p=.052]$. Mean Fisher's $z$-transformed correlation coefficients for ROI pairs that showed a reliable main effect of capacity are depicted as a function of reading capacity in Figure 5.

Functional connectivity analyses extended the evidence that high- and low-capacity readers were differentially sensitive to the lexical frequency manipulation. This Reading capacity $x$ Lexical frequency interaction was reliable for functional connectivity between right inferior frontal and left inferior occipital regions $[F(1,17)=5.47, M S E=0.23]$, and between right parietal and right inferior occipital regions $[F(1,30)=4.57, M S E=0.26]$. Reading capacity $\times$ Lexical frequency interactions between left inferior frontal and left inferior occipital $[F(1,32)$ $=3.35, M S E=0.16, p=.077]$ and between left parietal and right inferior occipital $[F(1,33)=$ $4.05, M S E=0.24, p=.052]$ approached significance. Follow-up analyses between right inferior frontal and left inferior occipital regions revealed that high-capacity readers showed significant 
increases in functional connectivity with decreasing lexical frequency $[F(1,7)=15.63, M S E$ $=0.17)$, whereas low-capacity readers showed no reliable effects of lexical frequency $(F<1)$. Follow-up analyses between right parietal and right inferior occipital regions showed a slightly different pattern. High-capacity readers did not show significant effects of lexical frequency $(F<1)$ between these regions; however, low-capacity readers showed a significant decrease in functional connectivity as a function of decreasing lexical frequency $[F(1,15)=7.39$, $M S E=0.50]$. Therefore, high-capacity readers were able to either increase or maintain synchronization as a function of decreasing lexical frequency, whereas low-capacity readers either maintained or decreased synchronization as a function of decreasing lexical frequency.

Functional connectivity analyses also provided evidence that high- and low-capacity readers were differentially sensitive to syntactic complexity, consistent with existing behavioral results. This Reading capacity $\times$ Syntax interaction was reliable between left inferior frontal and parietal regions $[F(1,32)=7.73, M S E=0.22]$ and approached significance between left temporal and left inferior occipital $[F(1,32)=3.9, M S E=0.19, p=.057]$ and between right inferior frontal and right temporal $[F(1,17)=4.14, M S E=0.06, p=.058]$ regions. Follow-up analyses revealed that high-capacity readers showed significantly increased functional connectivity as a function of increased syntactic complexity $[F(1,16)=15.19, M S E=0.20]$, whereas low-capacity readers did not show modulations in functional connectivity as a function of syntactic complexity $[F(1,16)=1.10, M S E=0.05, p>.30]$. Therefore, as the syntactic processing demand increased, high-capacity readers showed increased synchronization between cortical regions in the language network, whereas low-capacity readers showed no change in synchronization. Mean Fisher's $z$-transformed correlation coefficients for ROI pairs that showed reliable interactions between reading capacity and lexical frequency or between reading capacity and syntactic complexity are depicted as a function of reading capacity in Figure 6.

This experiment provided additional evidence that functional connectivity changes in the face of differing task demands. Across individuals, functional connectivity was higher for activeconjoined sentences than for object-relative sentences, resulting in a reliable main effect of syntactic complexity across most of the ROI pairs tested. Synchronization between left parietal and bilateral temporal regions was also higher for high-frequency words than for low-frequency words, resulting in a reliable main effect of lexical frequency. In addition, Syntactic complexity $\times$ Lexical frequency interactions were observed throughout many of the ROI pairs tested. In the high-frequency conditions, functional connectivity tended to be highest for active sentences; however, in the low-frequency conditions, functional connectivity was highest for object-relative sentences. This interaction was significant for functional connectivity between a number of ROI pairs, primarily those involving left parietal and inferior occipital regions. Reliable $F$ statistics are reported in Table 4.

\section{DISCUSSION}

The results of this study suggest that individual differences in reading ability are characterized by multiple dimensions of brain function. These findings are consistent with previous research suggesting that skilled performance is associated with more efficient neural systems. Across conditions, high-capacity readers showed lower levels of activation than did low-capacity readers, especially in the bilateral middle frontal gyri and right lingual gyrus. This suggests that high-capacity readers did not draw as heavily upon frontal planning/strategic networks and occipital word recognition areas as low-capacity readers did. Although they utilized fewer neural resources, high-capacity readers were faster and more accurate during the sentence comprehension task. Thus, the evidence suggests that high-capacity readers' processing was more efficient (used fewer resources) overall than was low-capacity readers'. 
In addition, this study extends previous research by exploring individual differences in neural adaptability. The results of this investigation suggest that the neural systems of high-capacity readers were more adaptable to changing task demands. ROI analyses revealed that highcapacity readers differentially activated left hemisphere language regions as a function of lexical frequency, whereas low-capacity readers did not. In other words, high-capacity readers more readily modulate the amount of neural resources that they utilize in the face of changing lexical demands than do low-capacity readers.

Functional connectivity analyses provided a critical link between comprehension ability and neural function, focusing on network-level properties rather than the property of any isolated cortical area. One striking result is that high-capacity readers have better synchronization across conditions between the traditional left hemisphere language regions, including Broca's and Wernicke's areas, than do low-capacity readers. This provides evidence that neural synchronization is systematically linked to language capacity. Such synchronization may reflect greater coordination or cooperation between regions among high-capacity readers. This finding does not, however, imply that the group differences are due to differences in anatomical connectivity. In fact, important interactions between reading capacity and both lexical and syntactic demands were observed, suggesting that group differences in functional connectivity are not completely explained by static differences in anatomical connectivity. As demand of any type increased, the neural networks of high-capacity readers were able to either maintain or increase synchronization. In contrast, the neural networks of low-capacity readers either remained constant or became less functionally coordinated as demands increased.

We interpret the relative differences found in functional connectivity as suggestive of differences in inter-regional communication, coordination, or cooperation. We note, however, that the operational definition of functional connectivity used in the current study is only one of many that have been used in the rapidly expanding literature (see Horwitz, 2003, for a review), and that because functional connectivity is a description of the correlation in the temporal dynamics of activation between regions, other interpretations of the linkage to reading capacity are possible. For example, the left hemisphere language areas of high-capacity readers might respond more similarly to a common linguistic input than those of low-capacity readers, and such a difference could drive the correlation between the activation time series in the absence of direct communication between the regions. However, the known anatomical connections among left hemisphere language areas and the necessity for integration of information computed in different regions for sentence comprehension make such interpretations less plausible. Future individual differences investigations using analysis techniques that examine effective connectivity (defined as the influence one region has on another; Friston, 1994), such as structural equation modeling (McIntosh \& Gonzalez-Lima, 1994) or dynamic causal modeling (Friston, Harrison, \& Penny, 2003), may provide additional insight into the causal mechanisms behind the differences in functional connectivity found here.

In this experiment, reading capacity was not only characterized by baseline differences in neural efficiency and synchronization. Rather, neural adaptability, as discussed above, may necessarily involve synchronization in order to function optimally. We propose that optimal cognitive functioning may require recruitment AND synchronization of brain regions in the face of increasing task demands. This combination of adaptability and synchronization appears to be a hallmark of comprehension skill. Consequently, this study illustrates the utility of functional connectivity as a potential tool for indexing individual differences in task proficiency. Moreover, it illustrates that individual differences need not be localized to a particular node in the cortical network, but to the nature of the co-functioning of two or more nodes. 
The individual differences measure used in this experiment, reading span, is a measure of verbal working memory capacity. Reading span scores are reliably highly correlated both with specific linguistic skills (e.g., making inferences, detecting ambiguity) and with more generalized tests of reading comprehension ability (e.g., Verbal Scholastic Aptitude Test, Nelson-Denny Reading Test) across a variety of experiments (see Daneman \& Merikle, 1996, for a meta-analysis). Working memory measures, in general, correlate quite well with measures of reasoning ability (Kyllonen \& Christal, 1990) and general fluid intelligence (Engle, Tuholski, Laughlin, \& Conway, 1999), even when crystallized intelligence is controlled for (Buehner, Krumm, Ziegler, \& Pluecken, 2006). Therefore, our characterization of skilled neural systems in individuals with high working memory capacity may ultimately be generalizeable to individual differences in reading ability and general intelligence measures.

This experiment represents the first attempt at characterizing the individual differences inherent in skilled neural systems. Previous neuroimaging investigations of individual differences have focused primarily on neural efficiency of specific brain regions. We argue that an accurate characterization of the neural basis of individual differences in cognition must include systemlevel descriptions. Given the complexities inherent in brain functioning and the multiple dimensions in which any individual may be skilled, it is not surprising that no single neural variable can explain the observed behavior differences in sentence comprehension.

\section{Acknowledgements}

This research was supported by the National Institute of Mental Health Grant no. MH029617 and by the National Institutes of Health Grant no. T32 MH19102.

\section{References}

Bell LC, Perfetti CA. Reading skill: Some adult comparisons. Journal of Educational Psychology 1994;86:244-255.

Boivin MJ, Giordani B, Berent S, Amato DA, Lehtinen S, Koepp RA, et al. Verbal fluency and positron emission tomographic mapping of regional cerebral glucose metabolism. Cortex 1992;28:231-239. [PubMed: 1499309]

Buchel C, Coull JT, Friston K. The predictive value of changes in effective connectivity for human learning. Science 1999;283:1538-1541. [PubMed: 10066177]

Buehner M, Krumm S, Ziegler M, Pluecken T. Cognitive abilities and their interplay: Reasoning, crystallized intelligence, working memory components, and sustained attention. Journal of Individual Differences 2006;27:57-72.

Caplan D, Alpert N. Effects of syntactic structure and propositional number on patterns of regional cerebral blood flow. Journal of Cognitive Neuroscience 1998;10:541-553. [PubMed: 9712683]

Daneman M, Carpenter PA. Individual differences in working memory and reading. Journal of Verbal Learning and Verbal Behavior 1980;19:450-466.

Daneman M, Merikle PM. Working memory and language comprehension: A meta-analysis. Psychonomic Bulletin \& Review 1996;3:422-433.

Diwadkar VA, Carpenter PA, Just MA. Collaborative activity between parietal and dorso-lateral prefrontal cortex in dynamic spatial working memory revealed by fMRI. Neuroimage 2000;12:85-99. [PubMed: 10875905]

Engle RW, Tuholski SW, Laughlin JE, Conway ARA. Working memory, short-term memory, and general fluid intelligence: A latent variable approach. Journal of Experimental Psychology: General 1999;128:309-331. [PubMed: 10513398]

Friston KJ. Functional and effective connectivity in neuroimaging: A synthesis. Human Brain Mapping 1994;2:56-78.

Friston KJ, Harrison L, Penny WD. Dynamic causal modeling. Neuroimage 2003;19:1273-1302. [PubMed: 12948688] 
Friston KJ, Holmes AP, Worsley KJ, Poline J, Frith CD, Frackowiak RSJ. Statistical parametric maps in functional imaging: A general linear approach. Human Brain Mapping 1995;2:189-210.

Haier RJ, Siegel BV, Nuechterlein KH, Hazlett E, Wu JC, Paek J, et al. Cortical glucose metabolic rate correlates of abstract reasoning and attention studied with positron emission tomography. Intelligence 1988;12:199-217.

Horowitz B. The elusive concept of brain connectivity. Neuroimage 2003;19:466-470. [PubMed: 12814595]

Just MA, Carpenter PA. A capacity theory of comprehension: Individual differences in working memory. Psychological Review 1992;99:122-149. [PubMed: 1546114]

Just MA, Carpenter PA, Keller TA, Eddy WF, Thulborn KR. Brain activation modulated by sentence comprehension. Science 1996;274:114-116. [PubMed: 8810246]

Keller TA, Carpenter PA, Just MA. The neural bases of sentence comprehension: A fMRI examination of syntactic and lexical processing. Cerebral Cortex 2001;11:223-237. [PubMed: 11230094]

Kucera, H.; Francis, WN. Computational analysis of present-day American English. Providence, RI: Brown University Press; 1967.

Kyllonen PC, Christal RE. Reasoning ability is (little more than) working memory capacity? Intelligence 1990;14:389-433.

Larson GE, Haier RJ, LaCasse L, Hazen K. Evaluation of a "mental effort" hypothesis for correlations between cortical metabolism and intelligence. Intelligence 1995;21:267-278.

Maxwell AE, Fenwick PBC, Fenton GW, Dollimore J. Reading ability and brain function: A simple statistical model. Psychological Medicine 1974;4:274-280. [PubMed: 4427975]

McIntosh AR, Gonzalez-Lima F. Structural equation modeling and its application to network analysis in functional brain imaging. Human Brain Mapping 1994;2:2-22.

Neubauer AC, Grabner RH, Freudenthaler HH, Beckmann JF, Guthke J. Intelligence and individual differences in becoming neurally efficient. Acta Psychologica 2004;116:55-74. [PubMed: 15111230]

Newman, S.; Just, MA. The neural bases of intelligence: A perspective based on functional neuroimaging. In: Sternberg, RJ.; Pretz, J., editors. Cognition and intelligence: Identifying the mechanisms of the mind. New York: Cambridge University Press; 2005. p. 88-103.

Newman SD, Carpenter PA, Varma S, Just MA. Frontal and parietal participation in problem solving in the Tower of London: fMRI and computational modeling of planning and high-level perception. Neuropsychologia 2003;41:1668-1682. [PubMed: 12887991]

Oldfield RC. The assessment of analysis of handedness: The Edinburgh inventory. Neuropsychologia 1971;9:97-113. [PubMed: 5146491]

Otsuka Y, Osaka N. An fMRI study of verbal working memory: Effects of aging on ACC-PFC network connectivity. Japanese Journal of Physiological Psychology and Psychophysiology 2005;23:11-18.

Reichle ED, Carpenter PA, Just MA. The neural basis of strategy and skill in sentence picture verification. Cognitive Psychology 2000;40:261-295. [PubMed: 10888341]

Tzourio-Mazoyer N, Landeau B, Papathanassiou D, Crivello F, Etard O, Delcroix N, et al. Automated anatomical labeling of activations in SPM using a macroscopic anatomical parcellation of the MNI MRI single-subject brain. Neuroimage 2002;15:273-289. [PubMed: 11771995] 


\section{Anatomically Defined Regions of Interest}

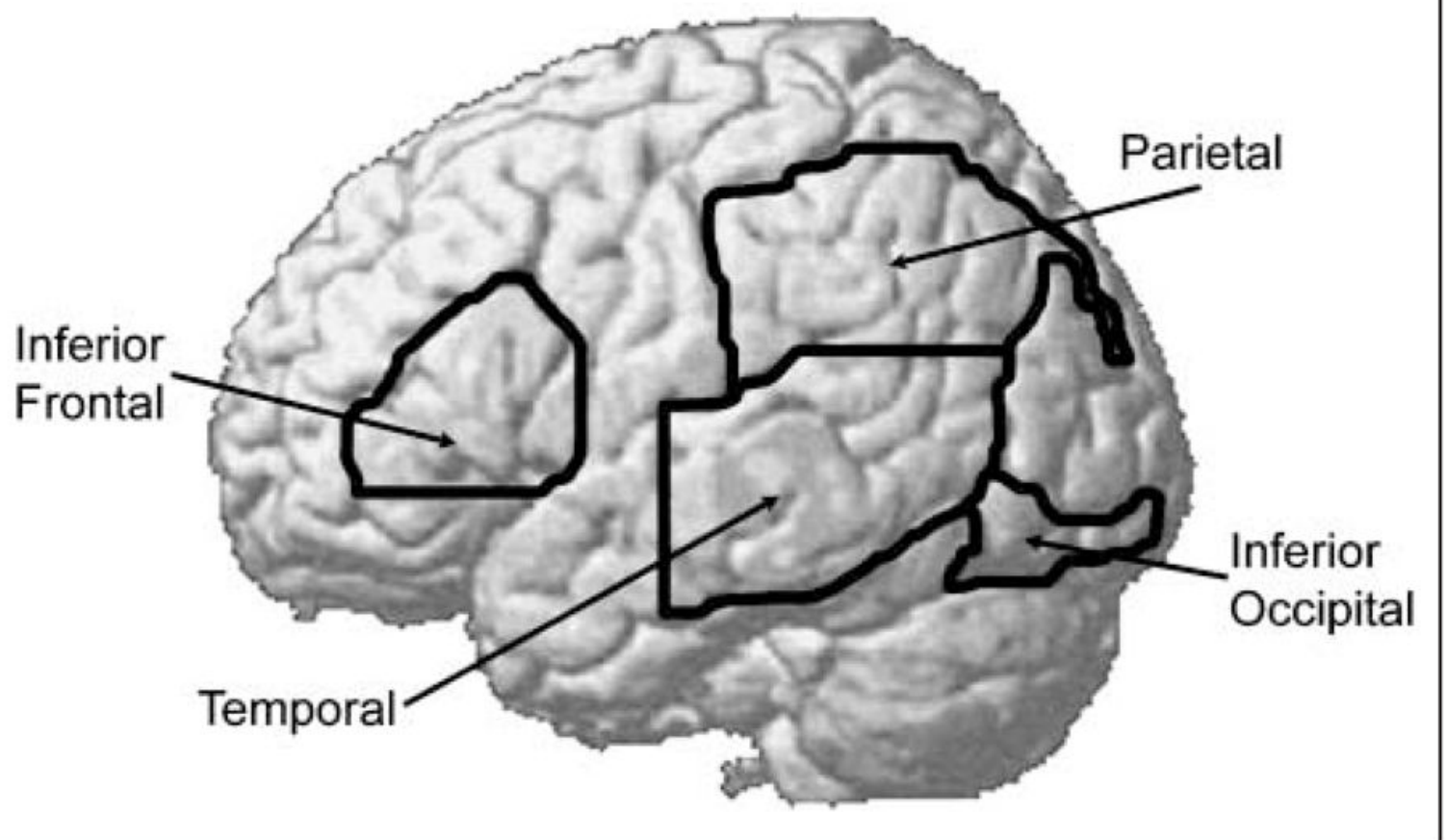

Figure 1.

Schematic depiction of the ROIs used for distribution of activation analyses. 


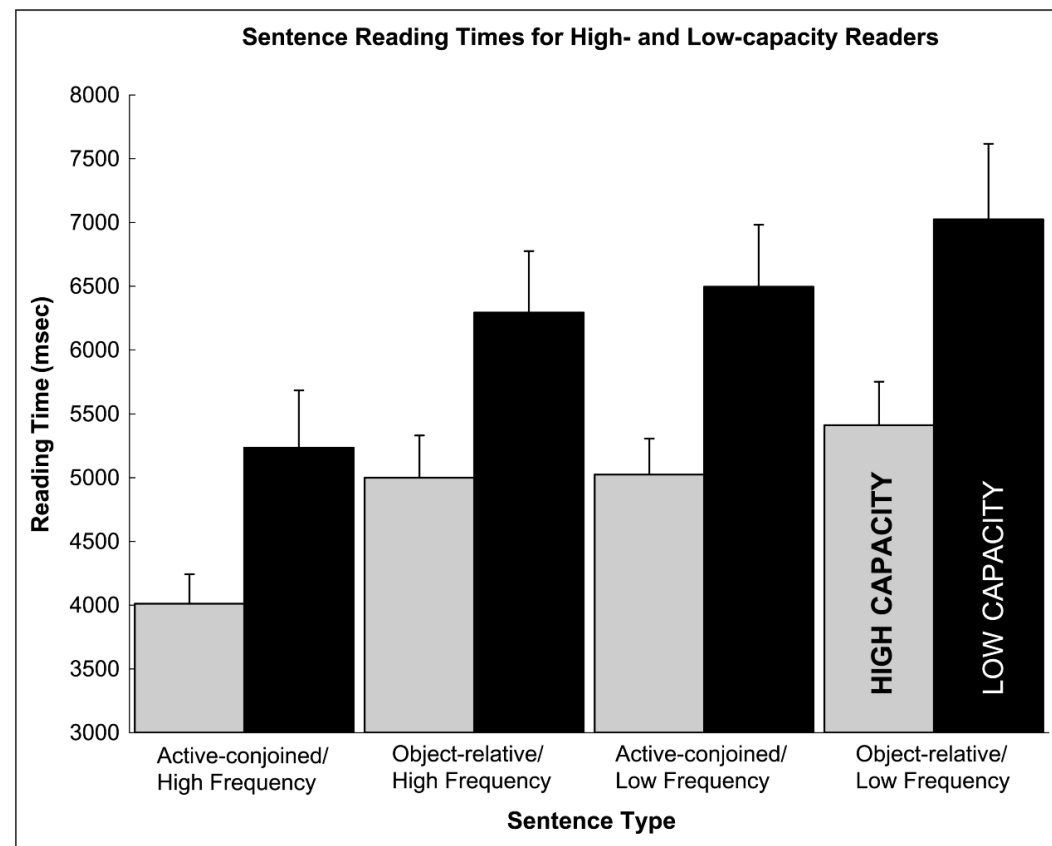

Mean Error Rates for High- and Low-capacity Readers

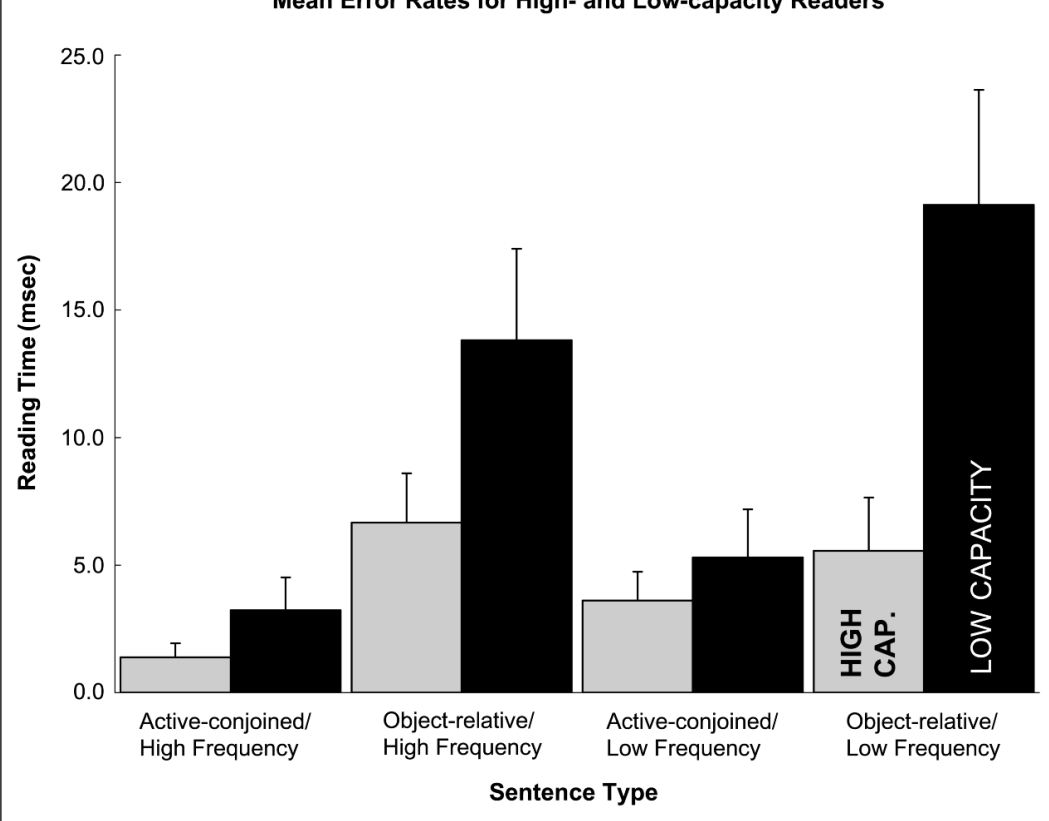

Figure 2.

Mean sentence reading times (msec) and error rates (\% error) as a function of reading capacity and sentence type. Error bars show the standard error of the mean. 


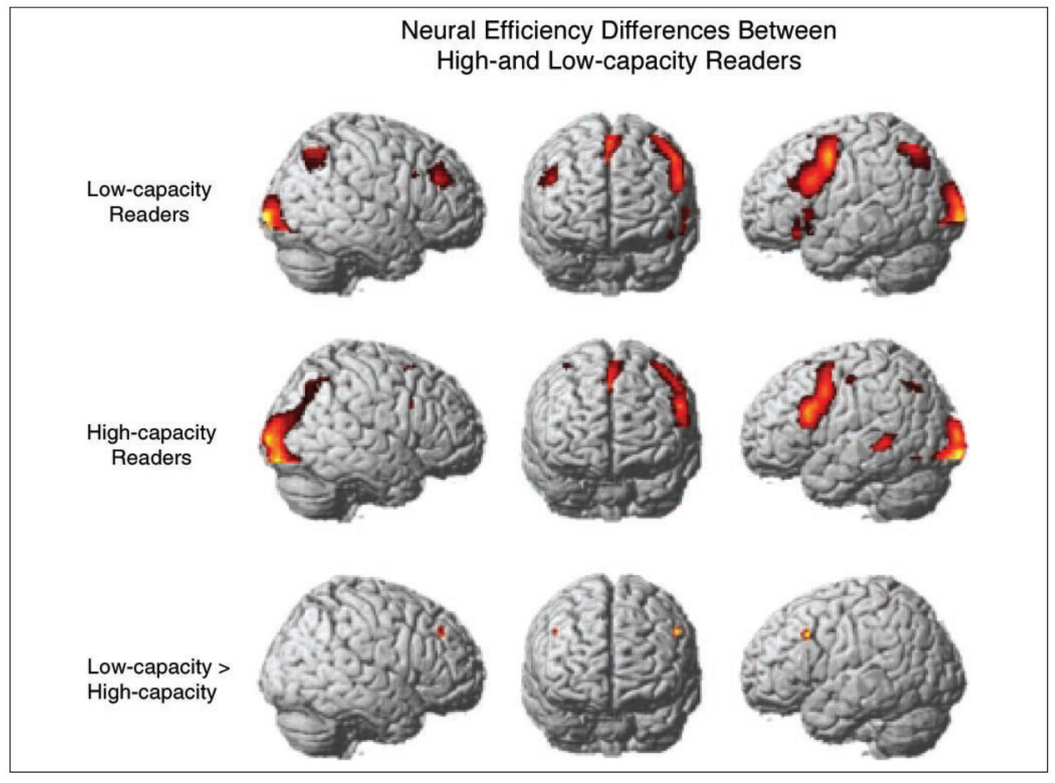

Figure 3.

Activation maps for high- and low-capacity readers as well as significant group differences based on voxelwise analyses. 


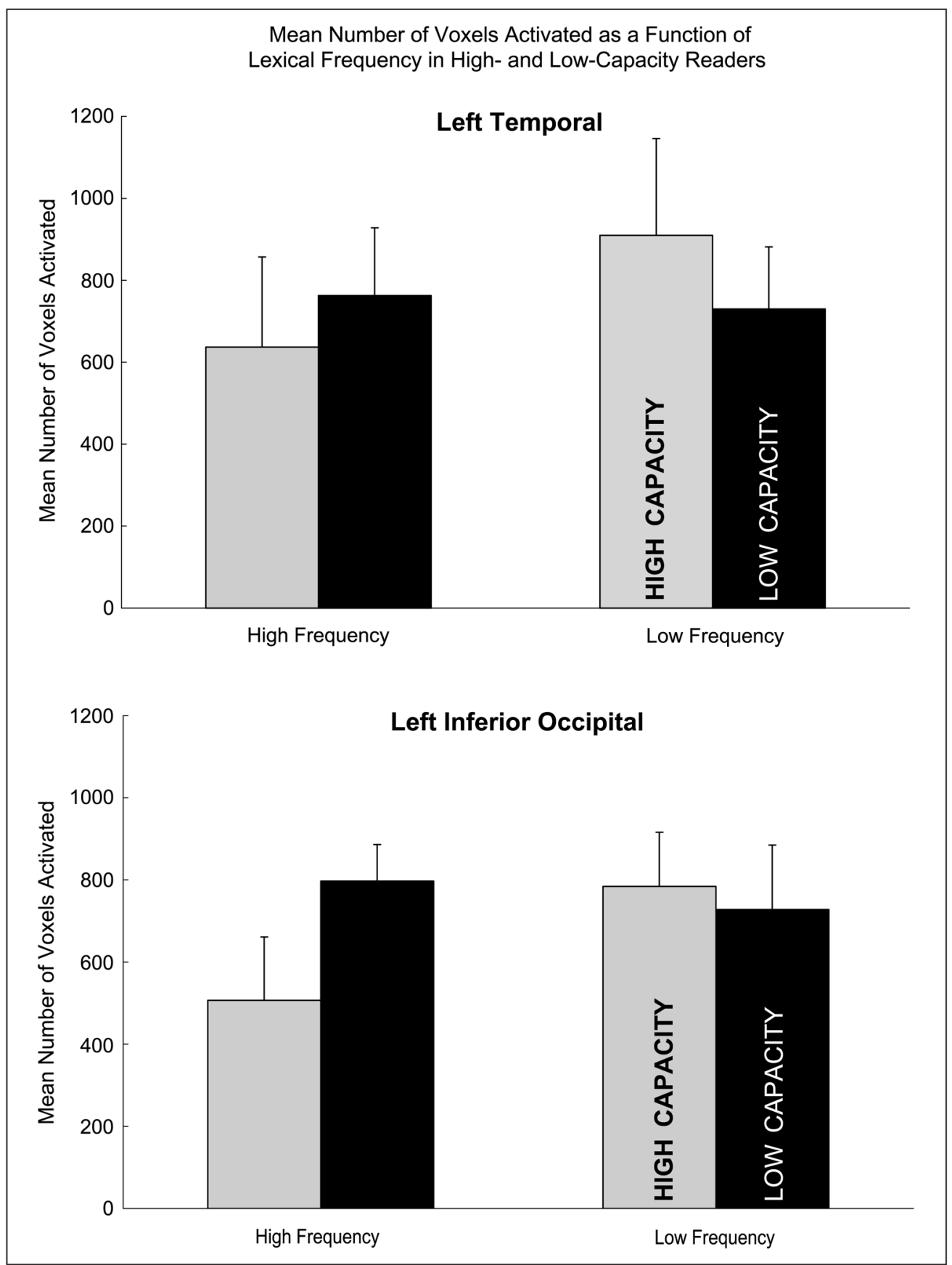

Figure 4.

Reading capacity $\times$ Lexical frequency interaction based on ROI analyses. Mean number of voxels activated in high- and low-capacity readers as a function of lexical frequency are depicted. Error bars show the standard error of the mean. 


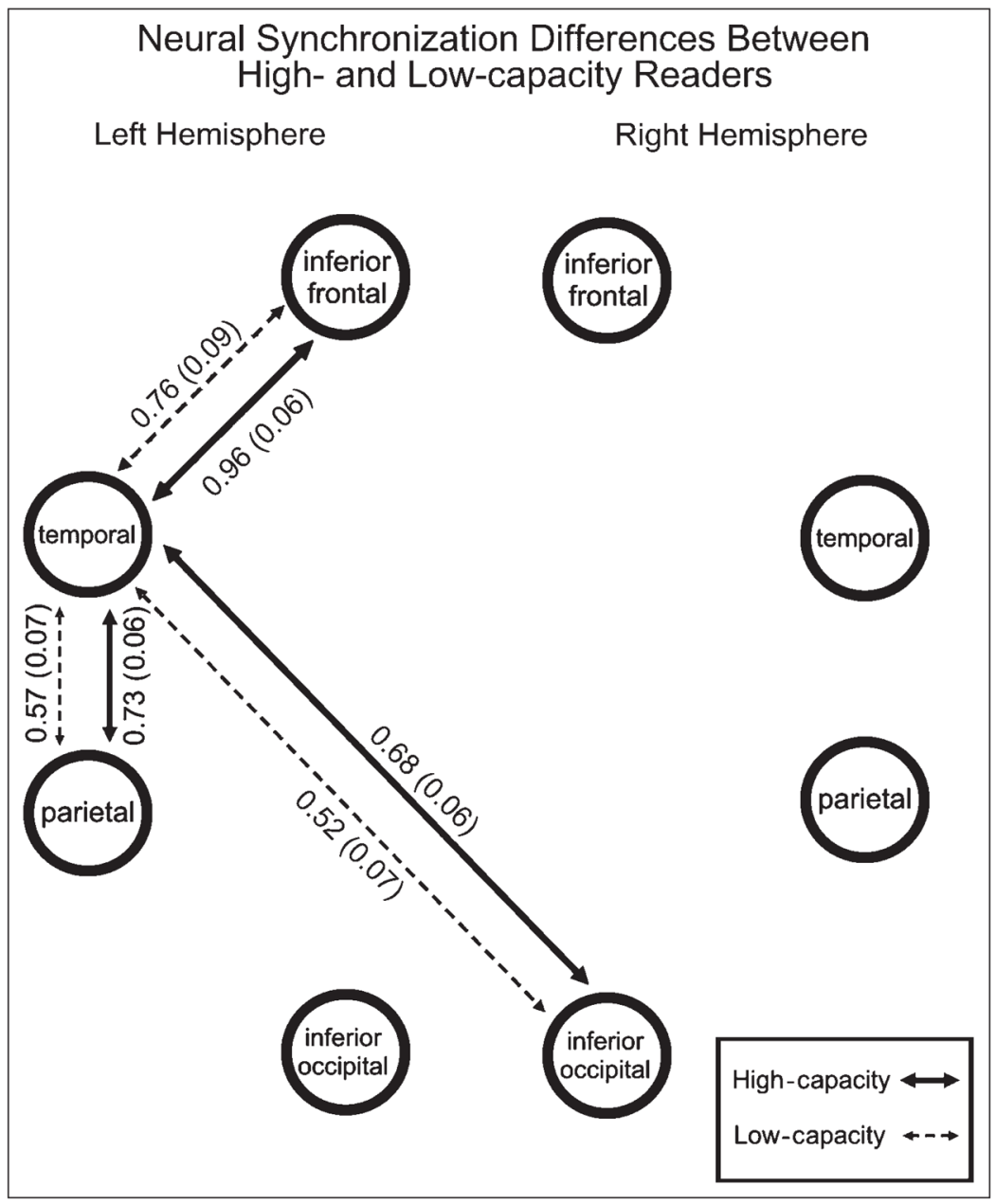

Figure 5.

Schematic depiction of ROI pairs with reliable main effects of reading capacity based on functional connectivity analyses. Mean functional connectivities (Fisher's $z$-transformed correlation coefficients) in high- and low-capacity readers are depicted (standard error of the mean in parentheses). 


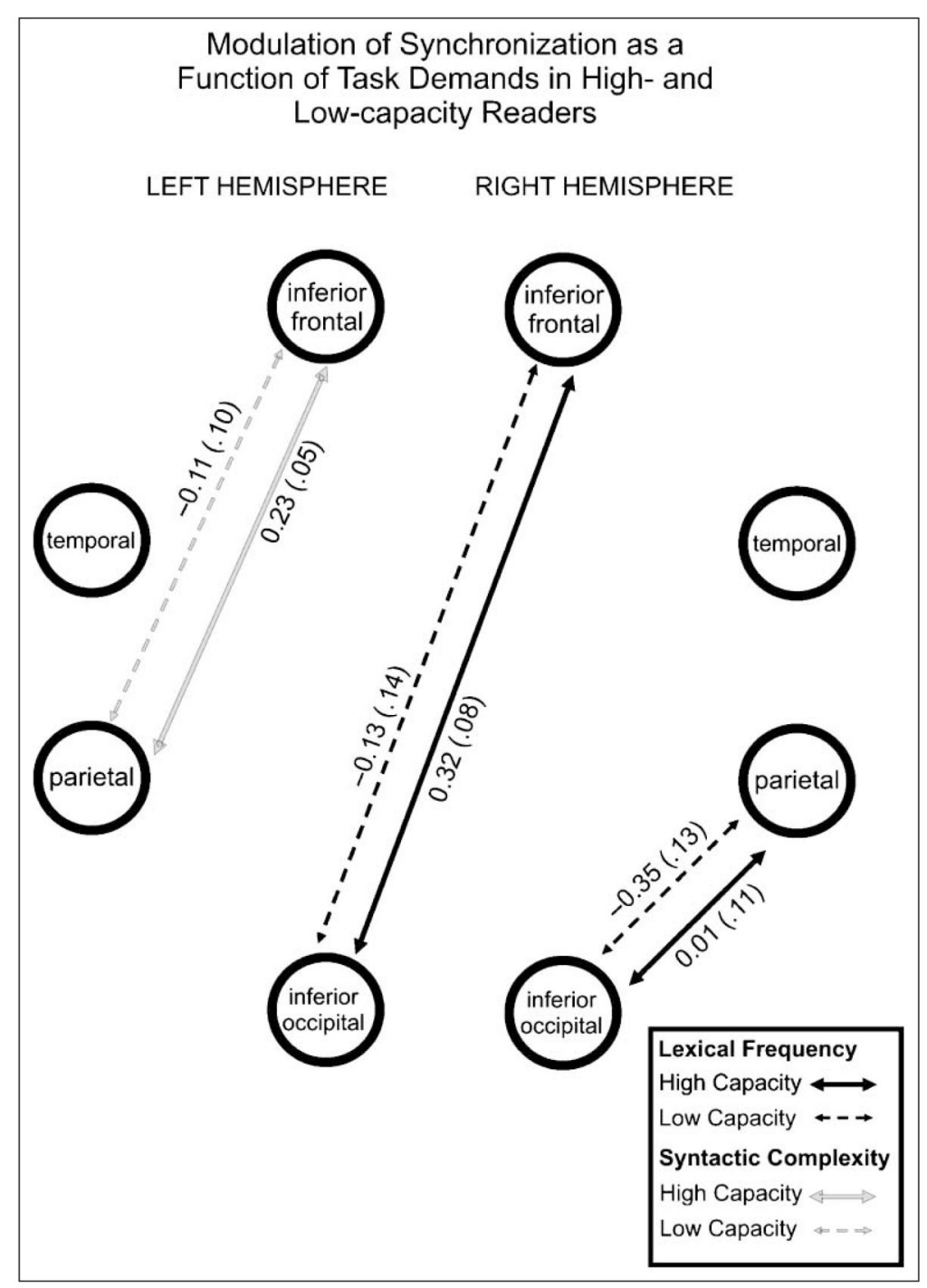

Figure 6.

Schematic depiction of ROI pairs with reliable Reading capacity $\times$ Lexical frequency (in black) and Reading capacity $\times$ Syntactic complexity (in gray) interactions based on functional connectivity analyses. Mean differences (Fisher's $z$-transformed correlation coefficients) between high- and low-demand conditions in high- and low-capacity readers are depicted (standard error of the mean in parentheses). 
Table 1

Sample Stimuli (from Keller et al., 2001)

High-frequency Nouns

Active-conjoined

The writer attacked the king and admitted the mistake at the meeting.

Object-relative

The writer that the king attacked admitted the mistake at the meeting. Low-frequency Nouns

Active-conjoined

The pundit attacked the regent and admitted the gaffe at the conclave.

Object-relative

The pundit that the regent attacked admitted the gaffe at the conclave. 
Prat et al.

Page 19

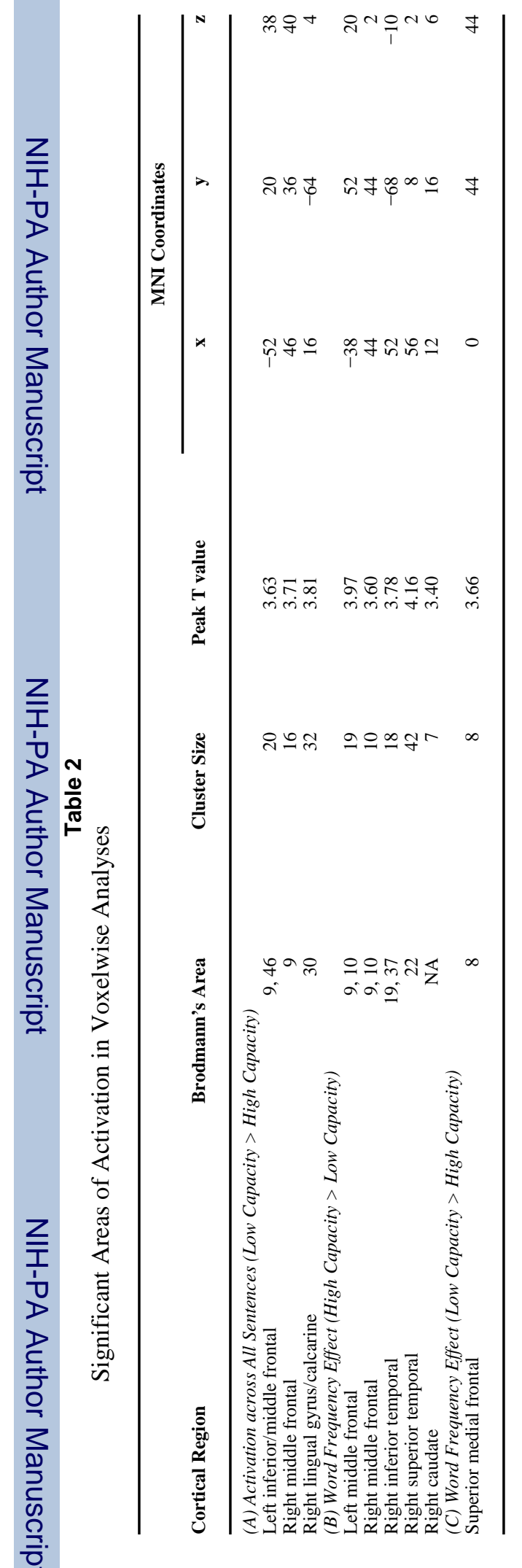

J Cogn Neurosci. Author manuscript; available in PMC 2008 December 10. 
Table 3

Mean Number of Voxels Activated for High- and Low-capacity Readers in the Four Sentence Types (Standard Errors of the Mean Appear in Parentheses)

\begin{tabular}{|c|c|c|c|c|}
\hline \multirow[b]{3}{*}{ ROI } & \multicolumn{4}{|c|}{ High-capacity Readers } \\
\hline & \multicolumn{2}{|c|}{ Active } & \multicolumn{2}{|c|}{ Object Relative } \\
\hline & High Frequency & Low Frequency & High Frequency & Low Frequency \\
\hline $\begin{array}{l}\text { Left inferior frontal } \\
\text { Left temporal } \\
\text { Left parietal } \\
\text { Left inferior occipital } \\
\text { Right inferior frontal } \\
\text { Right temporal } \\
\text { Right parietal } \\
\text { Right inferior occipital }\end{array}$ & $\begin{array}{c}199(44) \\
264(96) \\
288(60) \\
842(138) \\
60(31) \\
8(5) \\
206(64) \\
725(93)\end{array}$ & $\begin{array}{c}331(75) \\
401(112) \\
332(67) \\
1003(124) \\
114(59) \\
27(24) \\
214(75) \\
831(112)\end{array}$ & $\begin{array}{c}308(45) \\
373(124) \\
307(52) \\
834(119) \\
66(24) \\
10(6) \\
152(51) \\
691(89)\end{array}$ & $\begin{array}{c}453(79) \\
509(124) \\
457(80) \\
980(150) \\
109(48) \\
23(20) \\
237(70) \\
804(114)\end{array}$ \\
\hline \multirow{3}{*}{ Right inferior occipital } & \multicolumn{4}{|c|}{ Low-capacity Readers } \\
\hline & \multicolumn{2}{|c|}{ Active } & \multicolumn{2}{|c|}{ Object Relative } \\
\hline & High Frequency & Low Frequency & High Frequency & Low Frequency \\
\hline $\begin{array}{l}\text { Left inferior frontal } \\
\text { Left temporal } \\
\text { Left parietal } \\
\text { Left inferior occipital } \\
\text { Right inferior frontal } \\
\text { Right temporal } \\
\text { Right parietal } \\
\text { Right inferior occipital }\end{array}$ & $\begin{array}{c}351(84) \\
311(72) \\
395(70) \\
1181(143) \\
36(20) \\
45(38) \\
215(47) \\
1051(128)\end{array}$ & $\begin{array}{c}351(62) \\
354(67) \\
412(50) \\
1105(146) \\
80(35) \\
54(49) \\
241(49) \\
928(92)\end{array}$ & $\begin{array}{c}446(73) \\
452(94) \\
468(72) \\
1149(146) \\
55(32) \\
75(65) \\
219(48) \\
964(104)\end{array}$ & $\begin{array}{c}377(70) \\
376(84) \\
525(94) \\
1050(138) \\
49(22) \\
52(50) \\
245(56) \\
999(101)\end{array}$ \\
\hline
\end{tabular}


Table 4

ANOVA Statistics for Significant Functional Connectivity Analyses, by ROI

\begin{tabular}{|c|c|c|c|}
\hline ROI Pair & df & $\mathbf{F}$ & MSE \\
\hline \multicolumn{4}{|l|}{ (1) Main Effect of Syntax } \\
\hline Left inferior occipital-Left parietal & 1,33 & 17.41 & 0.62 \\
\hline Left inferior occipital-Right inferior occipital & 1,33 & 24.65 & 0.63 \\
\hline Left inferior occipital-Right parietal & 1,30 & 24.14 & 0.53 \\
\hline Left parietal-Right inferior occipital & 1,33 & 17.77 & 0.51 \\
\hline Left parietal-Right parietal & 1,30 & 16.94 & 0.30 \\
\hline Left temporal-Right inferior occipital & 1,32 & 13.17 & 0.43 \\
\hline Left temporal-Right parietal & 1,29 & 6.28 & 0.23 \\
\hline Left temporal-Right temporal & 1,10 & 8.77 & 0.29 \\
\hline Right inferior occipital-Right parietal & 1,30 & 16.22 & 0.51 \\
\hline Right inferior occipital-Right temporal & 1,10 & 6.02 & 0.20 \\
\hline Right parietal-Right inferior frontal & 1,9 & 7.37 & 0.16 \\
\hline \multicolumn{4}{|l|}{ (2) Main Effect of Lexical Frequency } \\
\hline Left temporal-Left parietal & 1,32 & 5.17 & 0.24 \\
\hline Right temporal-Left parietal & 1,10 & 9.87 & 0.16 \\
\hline \multicolumn{4}{|l|}{ (3) Frequency $\times$ Syntax Interactions } \\
\hline Left inferior occipital-Left parietal & 1,33 & 33.84 & 1.19 \\
\hline Left inferior occipital-Left temporal & 1,32 & 21.22 & 1.04 \\
\hline Left inferior occipital-Left inferior frontal & 1,32 & 7.77 & 0.33 \\
\hline Left inferior occipital-Right inferior occipital & 1,33 & 28.11 & 0.50 \\
\hline Left inferior occipital-Right parietal & 1,30 & 21.23 & 0.50 \\
\hline Left inferior occipital-Right inferior frontal & 1,17 & 16.81 & 0.65 \\
\hline Left parietal-Right inferior occipital & 1,33 & 22.99 & 0.84 \\
\hline Left parietal-Right parietal & 1,30 & 10.63 & 0.23 \\
\hline Left parietal-Right inferior frontal & 1,17 & 4.93 & 0.17 \\
\hline Left temporal-Right inferior occipital & 1,32 & 13.62 & 0.71 \\
\hline Left temporal-Right parietal & 1,29 & 5.98 & 0.23 \\
\hline Left temporal-Right temporal & 1,10 & 5.53 & 0.23 \\
\hline Left inferior frontal-Right inferior occipital & 1,32 & 6.10 & 0.25 \\
\hline Left inferior frontal-Right temporal & 1,29 & 9.32 & 0.22 \\
\hline Left inferior frontal-Right inferior frontal & 1,10 & 6.22 & 0.09 \\
\hline Right inferior occipital-Right Parietal & 1,30 & 21.65 & 0.44 \\
\hline Right parietal-Right temporal & 1,17 & 14.34 & 0.52 \\
\hline Right temporal-Right inferior frontal & 1,17 & 12.18 & 0.33 \\
\hline
\end{tabular}

